\title{
Prevention of calcium-induced membrane structural alterations in erythrocyte membranes by flunarizine
}

\author{
Peter G. Thomas ${ }^{a}$, Albert G. Zimmermann ${ }^{\text {b }}$ and A.J. Verkleij ${ }^{\text {b }}$ \\ a Institute of Molecular Biology and Medical Biotechnology and ${ }^{b}$ Department of Molecular Cell Biology. \\ University of Utrecht, Utrecht (The Netherlands)
}

(Received 17 August 1988)

\begin{abstract}
Key words: Calcium antagonist; Flunarizine; Calcium overload; Membrane structural organization; Freeze-fracture; Electron microscopy; (Human erythrocyte membrane)
\end{abstract}

The calcium antagonist flunarizine is shown to be able to prevent particle aggregation, membrane aggregation and blebbing resulting Irom elevated calcium concentrations. The anti-ischemic effects of flunarizine may therefore result in part from its ability to directly interfere with calcium-membrane interactions and thus prevent the lethal membrane reorganizations which occur after a period of ischemia during intracellular calcium overload.

Calcium plays an important, role as a second messenger in the function of many cell types, particularly in excitable tissues, by virtue of the discrepancy in its intra- and extracellular concentrations (see Ref. 1 and references therein). Consequently, its cellular concentration is subject to very careful control. However, under ischemic conditions this ccntrol, along with many other aspects of metabolic control is lost (for review, see Ref. 2). Upon reperfusion of ischemic tissue this loss of control leads to a massive increase in intracellular calcium concentration, the 'calcium overload', which is thought to be instrumental in the dramatic disruption of the plasma membrane which has been observed in a variety of tissues [3-5]. Various metabolites have been proposed as possible causative agents of this membrane disruption. These are generally products of calciumstimulated lipolytic activity; lysophospholipids,

Correspondence: P.G. Thomas, Institute of Molecular Biology and Medical Biotechnology, University of Utrecht, Padualaan 8, 3584 CH Utrecht, The Netherlands. free fatty acids, arachidonic acid and acyl carnitines (see Ref. 6 for review). It has recently been proposed, however, that the calcium itself could be responsible for post-ischemic membrane breakdown [7].

Calcium has a proven strong interaction with and influence upon the behavior of membrane phospholipid constituents, particularly acidic phospholipids [8]. It is proposed that by inducing phase separations of acidic phospholipids, especially phosphatid"lserine ("idSer), during ischemia and post-ischemic calcium overload, the calcium destabilizes membrane organization resulting in particle aggregation, blebbing and ultimately membrane disruption [7].

Most pharmaceutical agents used to treat ischemic conditions, the calcium antagonists, are thought to function by preventing this uptake of calcium by the membrane channel proteins and are better known as 'calcium-channel blockers' [9]. However, one group of calcium antagonists, the diphenylpiperizines typified by flunarizine (1-cinnamyl-4-(di-p-fluorobenzhydryl)piperazine), despite blocking cellular calcium uptake apparently 
do not interfere directly with the cellular uptake of calcium by these so-called 'calcium slow channels' [10]. To date the site of action of these drugs remains unknown.

Due to their lipophilic cationic properties we thought it interesting to investigate whether this group of compounds could influence the interactions of calcium with membranes. It is demonstrated here that in addition to its established calcium uptake blocking activity flunarizine exerts an extra calcium antagonistic effect by inhibiting the interaction of calcium with membrane components, namely phospholipids, thereby preventing damagin? membrane reorganization in response to high calc um concentrations.

A freeze-fracture electron microscopy (FFEM) study of inside-out vesicles (IOVs) from human erythrocytes was carried out to examine the effects of calcium on membrane structural organization and the possible antagonism of flunarizine. IOVs were chosen as a substitute for the cardiac sarcolemma for the following reasons. To date, it has not proven possible to have a good 'one-sided' sarcolemmal preparation in necessary quantity. It has, however, been recently shown that the myocyte plasma membrane (sarcolemma) is very similar to the erythrocyte with regard to its phospholipid composition and asymmetric distribution [11] and so the erythrocyte provides a suitable alternative. IOVs were chosen in preference to the normal erythrocyte membrane firstly because PtdSer which is normally found in the inner leaflet of the membrane is present on the outside and so is readily accessible to calcium. This is not true for erythrocytes which require the use of membrane disrupting agents or ionophores in order to examine calcium effects. Secondly, IOVs are free of the enormous cytoskeletal restraints that exist in the erythrocyte membrane.

In this report we show that in the erythrocyte IOV morel system we are able to simulate postischemic membrane reorganization by simply raising the calcium concentration. Pretreatment of the IOVs with the type IV calcium antagonist flunarizine prevents these alterations in membrane structural organization.

IOVs were prepared essentially according to the method of Steck and Kant [12] from freshly drawn human blood using citrate as anticoagulant.
Erythrocytes were collected by centrifugation and washed three times with 5 volumes of $150 \mathrm{mM}$ $\mathrm{NaCl}, 10 \mathrm{mM}$ sodium phosphate buffer ( $\mathrm{pH} \mathrm{8.0)}$. All further steps were carried out on ice or at $4^{\circ} \mathrm{C}$. Ghosts were then prepared by suspension of the erythrocytes in 40 volumes of $5 \mathrm{mM}$ sodium phosphate buffer ( $\mathrm{pH} \mathrm{8.0)}$ and were washed three times in the same buffer. To remove extrinsic cytoskeletal protein ghosts were diluted to 40 volumes with $0.5 \mathrm{mM}$ sodium phosphate buffer (pH 8.0) and left to stand overnight (approx. 18-20 h). After pelleting ghosts were vesiculated by being passed three times through a 27 gauge needle. When necessary, IOVs were purified using the dextrose cushion method of Steck and Kant and used immediately for further treatments.

For treatment with calcium and/or flunarizine a basis buffer of $0.5 \mathrm{mM}$ Hepes (4-(2-hydroxylethyl)-1-piperazineethanesulphonic acid) ( $\mathrm{pH}$ 7.4) was used. In order to test the effects of calcium and the possible antagonistic effects of flunarizine the following experimental regime was carried out at room temperature $\left(20-22^{\circ} \mathrm{C}\right)$. Two of four samples of $1 \mathrm{~cm}^{3}$ packed pellets were resuspended

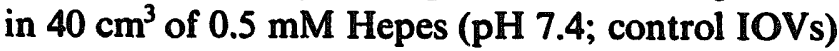
and the remaining two in $0.5 \mathrm{mM}$ Hepes containing $1 \mathrm{uM}$ flusiarizine (flunarizine IOVs) and left to stand for $1 \mathrm{~h}$ after which they were pelleted. One sample from each IOVs group was then resuspended in $0.5 \mathrm{mM}$ Hepes ( $\mathrm{pH} 7.4$ ) and the other in $0.5 \mathrm{mM}$ Hepes ( $\mathrm{pH}$ 7.4) containing $2 \mathrm{mM}$ $\mathrm{CaCl}_{2}$. They were again allowed to stand for $1 \mathrm{~h}$ and then pelleted prior to preparation for freezefracture electron microscopy (FFEM) examination. Incubation times of $1 \mathrm{~h}$ were used for both flunarizine and calcin treatments to ensure that a steady-state condition had been reached, which occurs after approx. 15 and $30 \mathrm{~min}$, respectively. This gives rise to four different samples: (i) Hepes/Hepes IOVs; (ii) Hepes/calcium IOVs; (iii) flunarizine/Hepes IOVs, and (iv) flunarizine/calcium IOVs. Samples were fast îrozen without cryoprotectant from $37^{\circ} \mathrm{C}$ in liquid propane using a Reichert-Jung KF80 freezing de-:ice and were freeze-fracture platinum-carbon replicated using either a Balzers' 300 or $400 \mathrm{~F}$ freezeetch apparatus. Replicas were cleaned using commercial bleach and examined using either a Philips 301 or 420 electron microscope. 

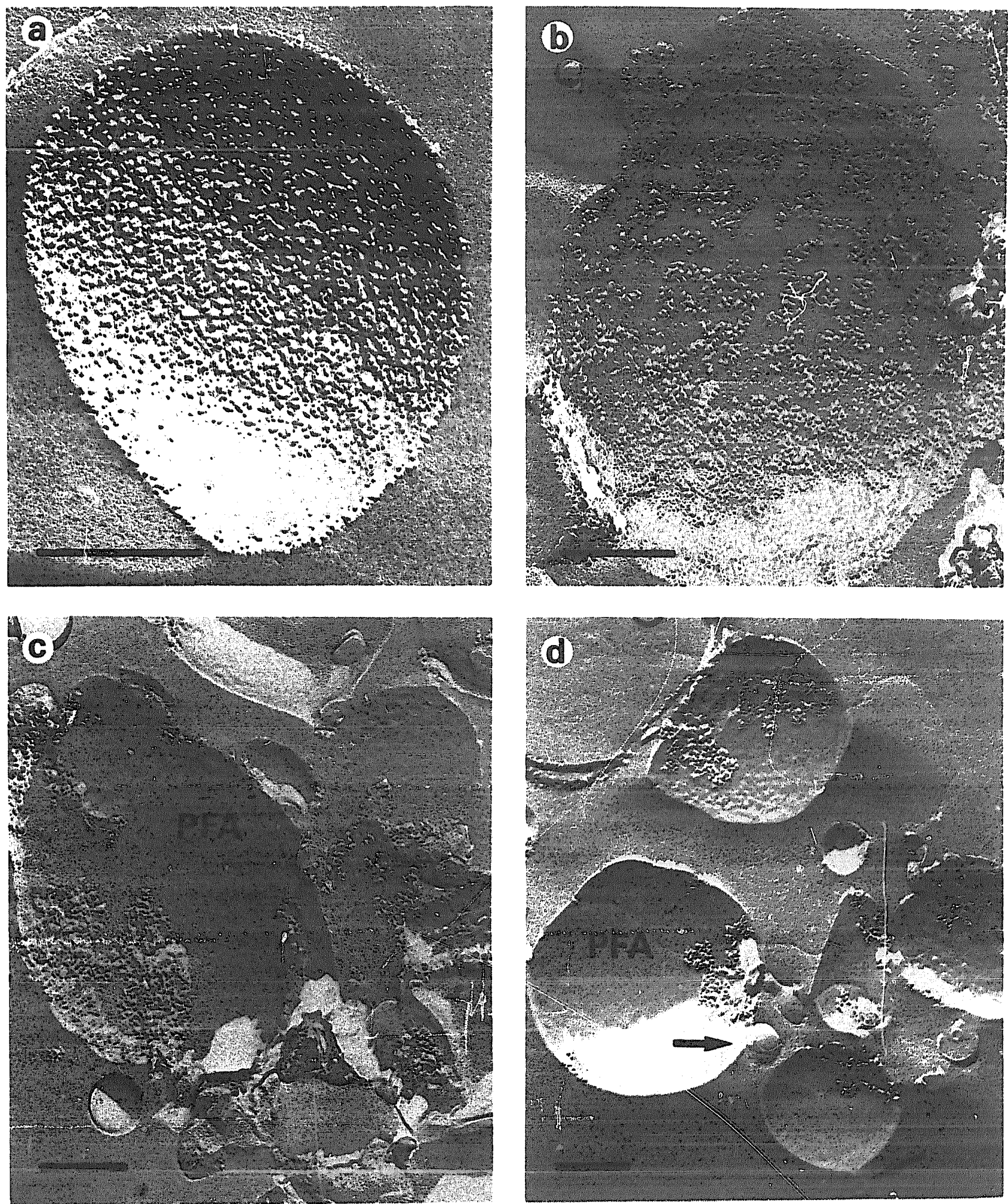

Fig. 1. Electron micrographs of freeze-fracture replicas of inside-out vesicles (IOVs) prepared from human erythrocytes (a) control, Hepes/Hepes IOVs and (b-d) Hepes/calcium JOVs following a 1 h treatment with $2 \mathrm{mM} \mathrm{Ca}^{2+}$. See text for further experimental details. Note that calcium treatment has resulted in particle-free areas (PFA), vesicle aggregation and blebbing of liposomal siructures (arrowed). Bars correspond to $250 \mathrm{~nm}$. 

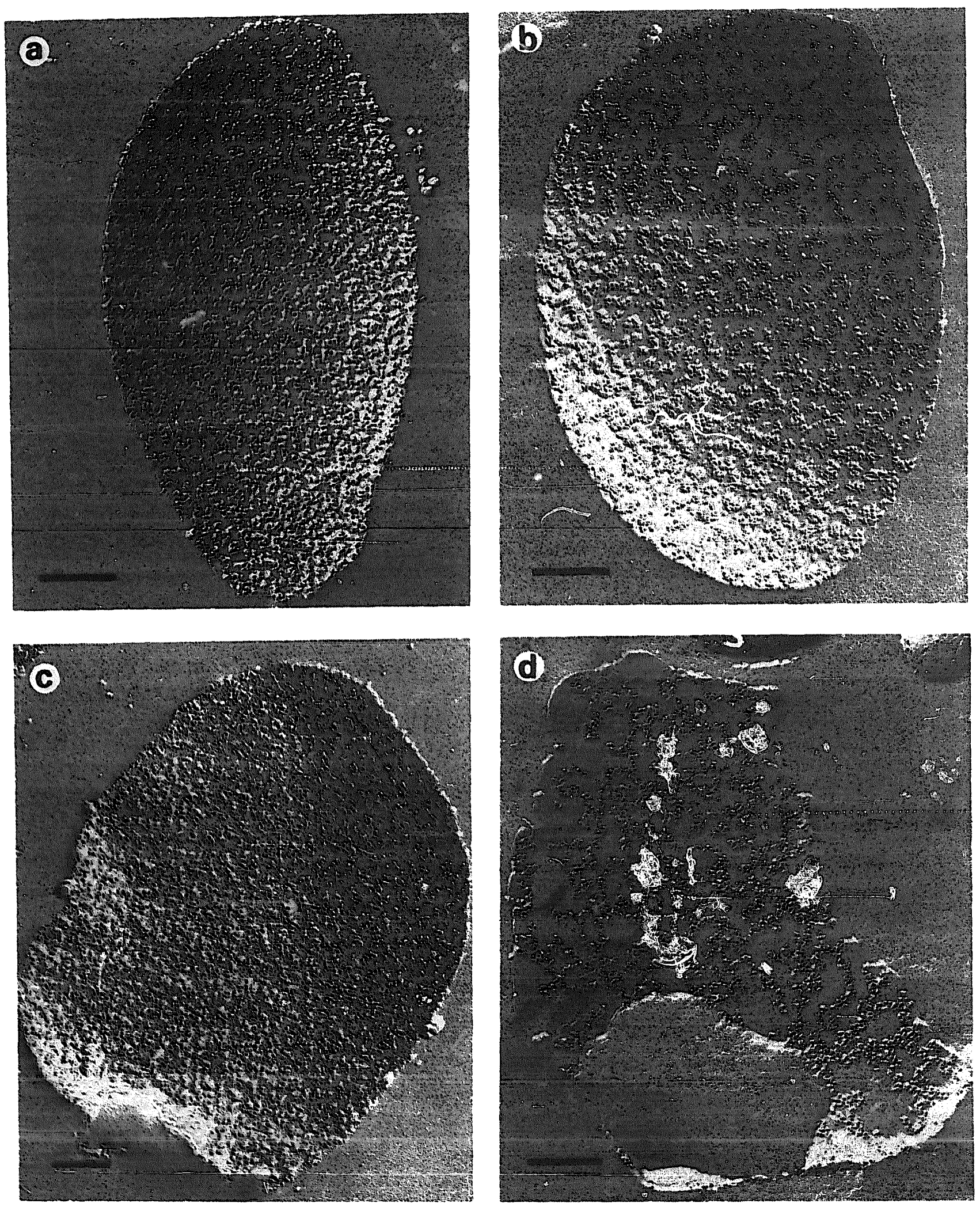

Fig. 2. Electron micrographs of freeze-fracture replicas of inside-out vesicles prepared from human erythrocytes and pre-incubated with $1 \mu \mathrm{M}$ flunarizine; (a and b). Control flunarizine/Hepes IOVs and (c and d) flunarizine/calcium IOVs following a $1 \mathrm{~h}$ treatment with $2 \mathrm{mM} \mathrm{Ca}^{2+}$. See text for further experimental details. Bars correspond to $250 \mathrm{~nm}$. 
Fig. 1a of control Hepes/Hepes IOVs confirms the inside-out orientation of the vesicles witnessed by the greater density of particles on the outer leaflet of the vesicles [13]. It can be clearly seen that the intramembrane particles (proteins) are evenly distributed over the surface of the membrane in this control sample. Examination of Hepes/calcium IOVs (Fig. 1b-d) shows that treatment with $2 \mathrm{mM}$ calcium has had a drastic effect on the distribution of the intramembrane particles. A range of effects can be seen from moderate lateral phase separation of membrane components (Fig. 1b), indicated by the 'netted' effect, to extreme lateral phase separation (Fig. 1c) shown by the large particle-free areas of membrane along with closely aggregated patches of intramembrane particles. This phenomenon has been seen previously with IOVs [13], but that study used much higher concentrations of calcium (10 $\mathrm{mM}$ ) and did not report the extreme particle aggregation shown here. Such lateral phase separation of membrane components is indicative of the transition of one of the phospholipid constituents from its liquid crystalline (fleid) phase to its gel (solid) phase [14]. In this case the isothermally calcium-induced phase transition of acidic phospholipids, namely PtdSer in which one calcium ion forms a complex with two lipid molecules [15]. Fig. 1d illusirates that under conditions of extreme lateral phase separation the blebbing of liposomal structures has occurred. These are identical in appearance to those seen in postischemically' reperfused cardiac tissue [16]. The above results support our proposal that the dramatic alterations observed in the plasma membrane of post-ischemically reperfused cardiac tissue could be caused by the calcium overload; a possible increase in cytoplasmic calcium concentration from $0.1-10 \mu \mathrm{M}$ to $1-2 \mathrm{mM}$ [1].

The diphenylpiperizine class of calcium antagonists are amphiphilic cationic amines and as such may have a similar inhibitory effect on the interaction of calcium with phospholipid inembranes as has been previously shown for certain local anesthetics [17]. In order to study this one group of $1 \bar{O} \overline{V s}$ was pretreated with $1 \mu \mathrm{M}$ flunarizine prior to exposure to calcium. Examination of flunarizine control IOVs (flunarizine/Hepes; Fig. 2a) shows that in the majority of vesicles pre-treat- ment of flunarizine has no discernable effect upon intramembrane particle distribution. Occasionally slight particle aggregation can be seen (Fig. 2b). It is perhaps to be expected that such a cationic amphiphile would have some effect on membrane constituent organization as has been previously observed with compounds such as acridine orange [18] and chlorpromazine [19]. Figs. 2c and 2d show the effects of exposure to $1 \mu \mathrm{M}$ flunarizine pre-treated IOVs to $2 \mathrm{mM}$ calcium (flunarizine/ calcium IOVs). In the majority of vesicles examined the calcium has had no effect at all on particle distribution. At most, slight lateral phase separation is visible in some cases. Thus, most dramatically, pre-treatment with $1 \mu \mathrm{M}$ flunarizine effectively prevents major calcium-induced membrane ultrastructural alterations.

Scheufler and co-workers have observed that under similar conditions approx. 1 mol flunarizine is present per $100 \mathrm{~mol}$ phospholipid bound to the membrane in the IOVs sample (personal communication). This would give a mole ratio of flunarizine to PtdSer of approximately $1: 13$ [21]. This suggests that relatively small quantities of flunarizine are able to influence the interaction of calcium with the membrane, thus preventing the calcium-induced lateral phase separations observed by freeze-fracture electron microscopy (FFEM). Recent work has also shown that flunarizine is also able to release calcium from PtsSer monolayers [20]. We are currently investigating the interactions of flunarizine and calcium with phospholipids in greater detail using several biophysical techniques.

In conclusion, we have shown that the erythrocyte membrane is a good model for the-myocyte plasma membrane with regard to simulating postischenuic alterations in membrane organization. The observations reported here using this system support the view that elevated calcium concentrations may cause or exacerbate the post-ischemic alterations in membrane structure. This model lends itself to studies of drug-membrane interactions and we have beeis able to observe the dramatic calcium antagonistic effects of flunarizine with regard to membrañe ultrastructural organization. The results demonstrate that flunarizine can indeed directly interfere with the interaction between calcium and membranes and 
is thereby able to prevent the deleterious effects of high calcium concentrations on membrane structure.

We gratefully acknowledge the financial assistance of Janssen Pharmaceutica B.V. (Tilburg, The Netherlands), and the scientific cooperation of the Janssen Research Foundation (Beerse, Belgium and Neuss, F.R.G.).

\section{References}

1 Cavero, I. and Spedding, M. (1983) Life Sci. 33, 2571-2581.

2 Van der Laarse, A. (1985) Mol. Physiol. 8, 577-591.

3 Coleman, S.E., Duggan, J. and Hackett, R.L. (1976) Lab. Invest. 35, 63-70.

4 Ashraf, M. and Halverson, C.A. (1977) Am. J. Pathol. 88, 583-594.

5 Farber, J.L., Martin, J.T. and Chien, K.R. (1978) Am. J. Pathol. 92, 713-732.

6 Sen, A., Buja, L.M., Willerson, J.T. and Chien, K.R. (1987) Basic Res. Cardiol. (Suppl.) 82, 121-125.

7 Verkleij, A.J. and Post, J.A. (1987) Basic Res. Cardiol. (Suppl.) 82, 85-91.

8 Seimiya, T. and Ohki, S. (1973) Biochim. Biophys. Acta 298, 546-561.
9 Spedding, M. and Cavero, I. (1984) Life Sci. 35, 575-587.

10 Holmes, B., Brogden, R.N., Heel, R.C., Speight, T.M. and Avery, C.S. (1984) Drugs 27, 6-44.

11 Post, J.A., Langer, G.A., Op den Kamp, J.A.F. and Verkleij, A.J. (1988) Biochim. Biophys. Acta 943, 256-266.

12 Steck, T.L. and Kant, J.A. (1974) Methods Enzymol. 31A, 172-180.

13 Gerritsen, W.J., Verkleij, A.J. and Van Deenen, L.L.M. (1978) Biochim. Biophys. Acta 555, 26-4i.

14 Verkleij, A.J. (1980) in Electron Microscopy at Molecular Dimensions (Baumeister, W. and Vogell, W., eds.), pp. 328-337, Springer Verlag, Berlin.

15 Portis, A., Newton, C., Pangborn, W. and Papahadjopoulos, D. (1979) Biochemistry 18, 780-790.

16 Post, J.A., Leunissen-Bijvelt, J., Ruigrok, T.J.C. and Verkleij, A.J. (1985) Biochim. Biophys. Acta 845, 119-123.

17 Cullis, P.R. and Verkleij, A.J. (1979) Biochim. Biophys. Acta 552, 546-551.

18 Lelkes, G., Lelkes, G., Szinyei Merse, K. and Hollan, S.R. (1983) Biochim. Biophys. Acta 732, 48-57.

19 Lieber, M.R., Lange, Y., Weinstein, R.S. and Steck, T.L. (1984) J. Biol. Chem. 259, 9225-9234.

20 Vogelgesang, R., Wood, G., Peters, G. and Scheufler, E. (1988) Biochem. Pharmacol. 37, 1597-1600.

21 Broekhuyse, R.M. (1969) Clin. Chim. A cta 23, 45\%-461. 